\title{
Derived categories for algebras with radical square zero
}

\author{
Viktor Bekkert and Yuriy Drozd \\ Dedicated to Ivan Shestakov for his 60th birthday.
}

\begin{abstract}
We determine the derived representation types of algebras with radical square zero and give a description of the indecomposable objects in their bounded derived categories.
\end{abstract}

\section{Introduction}

This paper is based on a talk given by the first author at the Conference "Algebras, Representations and Applications" (Sao Paulo, Brazil, August 2007).

Let $\mathcal{A}$ be a finite dimensional algebra over an algebraically closed field $\mathbb{k}, \mathcal{A}$-mod be the category of left finitely generated $\mathcal{A}$-modules and let $\mathcal{D}^{b}(\mathcal{A})$ be the bounded derived category of the category $\mathcal{A}$-mod.

The category $\mathcal{D}^{b}(\mathcal{A})$ is known for few algebras $\mathcal{A}$. For example, the structure of $\mathcal{D}^{b}(\mathcal{A})$ is well-known for hereditary algebras of finite and tame type $[\mathbf{H}]$ and for tubular algebras $\underline{\mathbf{H R}}$.

In the present paper we investigate the derived category $\mathcal{D}^{b}(\mathcal{A})$ for the finite dimensional algebras with radical square zero.

The structure of the paper is as follows. In Section 1 preliminary results about derived categories are given. We replace finite dimensional algebras by locally finite dimensional categories (shortly lofd). If such a category only has finitely many indecomposable objects, this language is equivalent to that of finite dimensional algebras.

In Section 2 for a given lofd category $\mathcal{A}$ with radical square zero, we construct following $[\mathbf{B D}$ a box such that its representations classify the objects of the derived category $\mathcal{D}^{b}(\mathcal{A})$, which is used in the next sections.

It follows from $[\mathbf{B D}$ that every lofd category over an algebraically closed field is either derived tame or derived wild. In Section 3 we establish the derived representation type for lofd categories with radical square zero.

2000 Mathematics Subject Classification. Primary 16G60, 16G70; Secondary 15A21, 16E05, $18 \mathrm{E} 30$.

Key words and phrases. Derived categories, algebras with radical square zero, derived representation type.

The first author was supported by FAPESP (Grant N 98/14538-0) and CNPq (Grant 301183/00-7). 
A description of indecomposables in $\mathcal{D}^{b}(\mathcal{A})$ is given in Section 4 Namely, we reduce this problem to the problem of description of indecomposables finite dimensional modules for some hereditary path algebra $\mathbb{k} \mathcal{Q}_{\mathcal{A}}$. In derived tame cases we describe indecomposables in $\mathcal{D}^{b}(\mathcal{A})$ explicitly.

After this paper was finished, we were told that similar results had been obtained by R. Bautista and S. Liu BL. Note that they use quite different methods.

\section{Derived categories}

We will follow in general the notations and terminology of [BD (see also [D3, D4).

We consider categories and algebras over a fixed algebraically closed field $\mathbb{k}$. A $\mathbb{k}$-category $\mathcal{A}$ is called locally finite dimensional (shortly lofd) if the following conditions hold:

1. All spaces $\mathcal{A}(x, y)$ are finite dimensional for all objects $x, y$.

2. $\mathcal{A}$ is fully additive, i.e. it is additive and all idempotents in it split. Conditions 1,2 imply that the category $\mathcal{A}$ is Krull-Schmidt, i.e. each object uniquely decomposes into a direct sum of indecomposable objects; moreover, it is local, i.e. for each indecomposable object $x$ the algebra $\mathcal{A}(x, x)$ is local. We denote by ind $\mathcal{A}$ a set of representatives of isomorphism classes of indecomposable objects from $\mathcal{A}$.

3. For each object $x$ the set $\{y \in$ ind $\mathcal{A} \mid \mathcal{A}(x, y) \neq 0$ or $\mathcal{A}(y, x) \neq 0\}$ is finite.

We denote by vec the category of finite dimensional vector spaces over $\mathbb{k}$ and by $\mathcal{A}$-mod the category of finite dimensional $\mathcal{A}$-modules, i.e. functors $M: \mathcal{A} \rightarrow$ vec such that $\{x \in \operatorname{ind} \mathcal{A} \mid M x \neq 0\}$ is finite.

For an arbitrary category $\mathcal{C}$ we denote by add $\mathcal{C}$ the minimal fully additive category containing $\mathcal{C}$. For instance, one can consider add $\mathcal{C}$ as the category of finitely generated projective $\mathcal{C}$-modules; especially, add $\mathbb{k}=$ vec. We denote by $\operatorname{Rep}(\mathcal{A}, \mathcal{C})$ the category of functors $\operatorname{Fun}(\mathcal{A}$, add $\mathcal{C})$ and call them representations of the category $\mathcal{A}$ in the category $\mathcal{C}$. Obviously, $\operatorname{Rep}(\mathcal{A}, \mathcal{C}) \simeq \operatorname{Rep}(\operatorname{add} \mathcal{A}, \mathcal{C})$. If the category $\mathcal{A}$ is lofd, we denote by $\operatorname{rep}(\mathcal{A}, \mathcal{C})$ the full subcategory of $\operatorname{Rep}(\mathcal{A}, \mathcal{C})$ consisting of the representations $M$ with finite support $\operatorname{supp} M=\{x \in$ ind $\mathcal{A} \mid M x \neq 0\}$. In particular, $\operatorname{rep}(\mathcal{A}, \mathbb{k})=\mathcal{A}$-mod.

We recall that a quiver is locally finite if at most finitely many arrows start or stop at each vertex. We recall also that every lofd category is equivalent to a quiver category, i.e. $\mathcal{A}=\operatorname{add} \mathbb{k} \mathcal{Q} / \mathcal{I}$, where $\mathcal{Q}=\mathcal{Q}_{\mathcal{A}}$ is the locally finite quiver of $\mathcal{A}$ and $\mathcal{I}$ is an admissible ideal in the path category $\mathbb{k} \mathcal{Q}$ of $\mathcal{Q}$.

We denote by $\mathcal{D}(\mathcal{A})$ (respectively, $\mathcal{D}^{b}(\mathcal{A})$ ) the derived category (respectively, (two-sided) bounded derived category) of the category $\mathcal{A}$-mod, where $\mathcal{A}$ is a lofd category. These categories are triangulated categories. We denote the shift functor by [1], and its inverse by [-1]. Recall that $\mathcal{A}^{\mathrm{op}}$ embeds as a full subcategory into $\mathcal{A}$-mod. Namely, each object $x$ corresponds to the functor $\mathcal{A}^{x}=\mathcal{A}\left(x,,_{-}\right)$. These functors are projective in the category $\mathcal{A}$-mod; if $\mathcal{A}$ is fully additive, these are all projectives (up to isomorphism). On the other hand, $\mathcal{A}$-mod embeds as a full subcategory into $\mathcal{D}^{b}(\mathcal{A})$ : a module $M$ is treated as the complex only having a unique nonzero component equal $M$ at the 0 -th position. It is also known that $\mathcal{D}^{b}(\mathcal{A})$ can be identified with the category $\mathcal{K}^{-, b}(\mathcal{A})$ whose objects are right bounded complexes of projective modules with bounded homology (that is, complexes of finitely generated projective modules with the property that the homology groups are non 
zero only at a finite number of places) and morphisms are homomorphisms of complexes modulo homotopy $\mathbf{G M}$. If $\operatorname{gl} \operatorname{dim} \mathcal{A}<\infty$, every bounded complex has a bounded projective resolution, hence $\mathcal{D}^{b}(\mathcal{A})$ can identified with $\mathcal{K}^{b}(\mathcal{A})$, the category of bounded projective complexes modulo homotopy, but that is not the case if gl. $\operatorname{dim} \mathcal{A}=\infty$. Moreover, if $\mathcal{A}$ is lofd, we can confine the considered complexes by minimal ones, i.e. always suppose that $\operatorname{Im} d_{n} \subseteq \operatorname{rad} P_{n-1}$ for all $n$. We denote by $\mathcal{P}_{\min }^{b}(\mathcal{A})$ the category of minimal bounded complexes of projective $\mathcal{A}$-modules.

Given $M \in \mathcal{D}^{b}(\mathcal{A})$, we denote by $P_{M}$ the minimal projective resolution of $M$.

For $P \neq 0 \in \mathcal{K}^{b}(\mathcal{A})$, let $t$ be the minimal number such that $P_{i}=0$ for $i>t$. Then, $\beta(P)$ denotes the (good) truncation of $P$ below $t$, i.e. the complex given by

$$
\begin{gathered}
\beta(P)_{i}= \begin{cases}P_{i} & , \text { if } i \leq t ; \\
\operatorname{Ker} d(P)_{t} & , \text { if } i=t+1 ; \\
0 & , \text { otherwise, }\end{cases} \\
d(\beta(P))_{i}= \begin{cases}d(P)_{i} & , \text { if } i \leq t ; \\
i_{\operatorname{Ker} d(P)_{t}} & , \text { if } i=t+1 ; \\
0 & , \text { otherwise }\end{cases}
\end{gathered}
$$

where $i_{\operatorname{Ker} d_{P}^{t}}$ is the obvious embedding.

Let $\overline{\mathcal{X}(\mathcal{A})}=\left\{M \in\right.$ ind $\left.\mathcal{P}_{\text {min }}^{b}(\mathcal{A}) \mid P_{\beta(M)} \notin \mathcal{K}^{b}(\mathcal{A})\right\}$. Let $\cong_{\mathcal{X}}$ be the equivalence relation on the set $\overline{\mathcal{X}(\mathcal{A})}$ defined by $M \cong \mathcal{X} N$ if and only if $P_{\beta(M)} \cong P_{\beta(N)}$ in $\mathcal{K}^{-, b}(\mathcal{A})$. We use the notation $\mathcal{X}(\mathcal{A})$ for a fixed set of representatives of the quotient set $\overline{\mathcal{X}(\mathcal{A})}$ over the equivalence relation $\cong{ }_{\mathcal{X}}$.

Proposition 1.1. [BM] ind $\mathcal{D}^{b}(\mathcal{A})=\operatorname{ind} \mathcal{P}_{\text {min }}^{b}(\mathcal{A}) \cup\{\beta(M) \mid M \in \mathcal{X}(\mathcal{A})\}$.

Remark 1.2. If $\mathcal{A}$ has finite global dimension, we have $\mathcal{X}(\mathcal{A})=\emptyset$ and hence ind $\mathcal{D}^{b}(\mathcal{A})=$ ind $\mathcal{P}_{\min }^{b}(\mathcal{A})$.

We recall the definitions of derived tame and derived wild lofd categories from BD.

Definition 1.3. 1. The rank of an object $x \in \mathcal{A}$ (or of the corresponding projective module $\mathcal{A}^{x}$ ) is the function $\mathbf{r}(x)$ : ind $\mathcal{A} \rightarrow \mathbb{Z}$ such that $x \simeq \bigoplus_{y \in \operatorname{ind} \mathcal{A}} \mathbf{r}(x)(y) y$. The vector rank $\mathbf{r}_{\bullet}\left(P_{\bullet}\right)$ of a bounded complex of projective $\mathcal{A}$-modules is the sequence $\left(\ldots, \mathbf{r}\left(P_{n}\right), \mathbf{r}\left(P_{n-1}\right), \ldots\right)$ (actually it has only finitely many nonzero entries).

2. We call a rational family of bounded minimal complexes over $\mathcal{A}$ a bounded complex $\left(P_{\bullet}, d_{\bullet}\right)$ of finitely generated projective $\mathcal{A} \otimes \mathrm{R}$-modules, where $\mathrm{R}$ is a rational algebra, i.e. $\mathrm{R}=\mathbb{k}\left[t, f(t)^{-1}\right]$ for a nonzero polynomial $f(t)$, and $\operatorname{Im} d_{n} \subseteq \mathrm{J} P_{n-1}$ For such a complex we define $P_{\bullet}(m, \lambda)$, where $m \in \mathbb{N}, \lambda \in \mathbb{k}, f(\lambda) \neq 0$, the complex $\left(P_{\bullet} \otimes_{\mathrm{R}} \mathrm{R} /(t-\lambda)^{m}, d_{\bullet} \otimes 1\right)$. It is indeed a complex of projective $\mathcal{A}$-modules. We put $\mathbf{r}_{\bullet}\left(P_{\bullet}\right)=\mathbf{r}_{\bullet}\left(P_{\bullet}(1, \lambda)\right)$ (this vector rank does not depend on $\lambda$ ).

3. We call a lofd category $\mathcal{A}$ derived tame if there is a set $\mathfrak{P}$ of rational families of bounded complexes over $\mathcal{A}$ such that:

(a) For each vector rank $\mathbf{r}_{\bullet}$ the set $\mathfrak{P}\left(\mathbf{r}_{\bullet}\right)=\left\{P_{\bullet} \in \mathfrak{P} \mid \mathbf{r}_{\bullet}\left(P_{\bullet}\right)=\mathbf{r}\right\}$ is finite. 
(b) For each vector rank $\mathbf{r}_{\bullet}$ all indecomposable complexes $\left(P_{\bullet}, d_{\bullet}\right)$ of projective $\mathcal{A}$-modules of this vector rank, except finitely many isomorphism classes, are isomorphic to $P_{\bullet}(m, \lambda)$ for some $P_{\bullet} \in \mathfrak{P}$ and some $m, \lambda$.

The set $\mathfrak{P}$ is called a parameterising set of $\mathcal{A}$-complexes.

4. We call a lofd category $\mathcal{A}$ derived wild if there is a bounded complex $P_{\bullet}$ of projective modules over $\mathcal{A} \otimes \Sigma$, where $\Sigma$ is the free $\mathbb{k}$-algebra in 2 variables, such that, for every finite dimensional $\Sigma$-modules $L, L^{\prime}$,

(a) $P_{\bullet} \otimes_{\Sigma} L \simeq P_{\bullet} \otimes_{\Sigma} L^{\prime}$ if and only if $L \simeq L^{\prime}$.

(b) $P \bullet \otimes_{\Sigma} L$ is indecomposable if and only if so is $L$.

(It is well-known that then an analogous complex of $\mathcal{A} \otimes \Gamma$-modules exists for every finitely generated $\mathbb{k}$-algebra $\Gamma$.)

Note that, according to these definitions, every derived discrete (in particular, derived finite) lofd category $[\mathbf{V}]$ is derived tame (with the empty set $\mathfrak{P}$ ).

It was proved in $\mathbf{B D}$ that every lofd category over an algebraically closed field is either derived tame or derived wild.

\section{Related boxes}

Recall (see [D1, D2]) that a box is a pair $\mathfrak{A}=(\mathcal{A}, \mathcal{V})$ consisting of a category $\mathcal{A}$ and an $\mathcal{A}$-coalgebra $\mathcal{V}$. We denote by $\mu$ the comultiplication in $\mathcal{V}$, by $\varepsilon$ its counit and by $\overline{\mathcal{V}}=\operatorname{ker} \varepsilon$ its kernel. We always suppose that $\mathfrak{A}$ is normal, i.e. there is a section $\omega: x \rightarrow \omega_{x}(x \in$ ob $\mathcal{A})$ such that $\varepsilon\left(\omega_{x}\right)=1_{x}$ and $\mu\left(\omega_{x}\right)=\omega_{x} \otimes \omega_{x}$ for all $x$. A category $\mathcal{A}$ is called free if it is isomorphic to a path category $\mathbb{k} \mathcal{Q}$ of a quiver $\mathcal{Q}$. A normal box $\mathfrak{A}=(\mathcal{A}, \mathcal{V})$ is called free if so is the category $\mathcal{A}$, while the kernel $\overline{\mathcal{V}}$ is a free $\mathcal{A}$-bimodule.

Recall that the differential of a normal box $\mathfrak{A}=(\mathcal{A}, \mathcal{V})$ is the pair $\partial=\left(\partial_{0}, \partial_{1}\right)$ of mappings, $\partial_{0}: \mathcal{A} \rightarrow \overline{\mathcal{V}}, \partial_{1}: \overline{\mathcal{V}} \rightarrow \overline{\mathcal{V}} \otimes_{\mathcal{A}} \overline{\mathcal{V}}$, namely

$$
\begin{gathered}
\partial_{0} a=a \omega_{x}-\omega_{y} a \text { for } a \in \mathcal{A}(x, y), \\
\partial_{1} v=\mu(v)-v \otimes \omega_{x}-\omega_{y} \otimes v \text { for } v \in \overline{\mathcal{V}}(x, y) .
\end{gathered}
$$

A representation of a box $\mathfrak{A}=(\mathcal{A}, \mathcal{V})$ over a category $\mathcal{C}$ is defined as a functor $M: \mathcal{A} \rightarrow$ add $\mathcal{C}$. A morphism of such representations $f: M \rightarrow N$ is defined as a homomorphisms of $\mathcal{A}$-modules $\mathcal{V} \otimes_{\mathcal{A}} M \rightarrow N$. If $g: N \rightarrow L$ is another morphism, there product is defined as the composition

$$
\mathcal{V} \otimes_{\mathcal{A}} M \stackrel{\mu \otimes 1}{\rightarrow} \mathcal{V} \otimes_{\mathcal{A}} \mathcal{V} \otimes_{\mathcal{A}} M \stackrel{1 \otimes f}{\rightarrow} \mathcal{V} \otimes_{\mathcal{A}} N \stackrel{g}{\rightarrow} L
$$

Thus we obtain the category of representations $\operatorname{Rep}(\mathfrak{A}, \mathcal{C})$. If $\mathfrak{A}$ is a free box, we denote by $\operatorname{rep}(\mathfrak{A}, \mathcal{C})$ the full subcategory of $\operatorname{Rep}(\mathfrak{A}, \mathcal{C})$ consisting of representations with finite support $\operatorname{supp} M=\{x \in \mathrm{ob} \mathcal{A} \mid M x \neq 0\}$. If $\mathcal{C}=$ vec, we write $\operatorname{Rep}(\mathcal{A})$ and $\operatorname{rep}(\mathcal{A})$.

Given a lofd $\mathcal{A}$ with radical square zero, we are going to construct a box such that its representations classify the objects of the derived category $\mathcal{D}^{b}(\mathcal{A})$ (see $\mathbf{B D}$, D3. for the case of an arbitrary lofd category).

Let $\mathcal{Q}=\mathcal{Q}_{\mathcal{A}}$ be a quiver of $\mathcal{A}$. Given two vertices $a$ and $b$ we define $\mathcal{Q}_{1}[a, b]$ as the set of all arrows from $a$ to $b$. Given an arrow $a$ of $\mathcal{Q}$, let us denote by $a^{-1}$ a formal inverse of $a$, and let us set $s\left(a^{-1}\right)=t(a)$ and $t\left(a^{-1}\right)=s(a)$. By a walk $w$ of length $n$ we mean a sequence $w_{1} w_{2} \cdots w_{n}$ where each $w_{i}$ is either of the form $a$ 
or $a^{-1}, a$ being an arrow in $\mathcal{Q}$ and where $s\left(w_{i+1}\right)=t\left(w_{i}\right)$ for $1 \leq i<n$. For each walk $w=w_{1} w_{2} \cdots w_{n}$ we define $s(w)=s\left(w_{1}\right)$ and $t(w)=t\left(w_{n}\right)$. By definition, a closed walk is a walk $w$ such that $s(w)=t(w)$.

Consider the path category $\mathcal{A}^{\square}=\mathbb{k} \mathcal{Q}^{\square}$, where $\mathcal{Q}^{\square}$ is the quiver with the set of points $\mathcal{Q}_{0}^{\square}=\mathcal{Q}_{0} \times \mathbb{Z}$ and with the set of arrows $\mathcal{Q}_{1}^{\square}=\mathcal{Q}_{1} \times \mathbb{Z}$, where for given $\alpha: a \rightarrow b$ in $\mathcal{Q}$ we set $s((\alpha, i))=(b, i)$ and $t((\alpha, i)):=(a, i-1)$.

Consider the normal free box $\mathfrak{A}=\mathfrak{A}(\mathcal{A})=\left(\mathcal{A}^{\square}, \mathcal{W}\right)$, with the kernel $\overline{\mathcal{W}}$ freely generated by the set $\left\{\varphi_{\alpha, i} \mid \alpha \in \mathcal{Q}_{1}, i \in \mathbb{Z}\right\}$, where $s\left(\varphi_{\alpha, i}\right)=(t(\alpha), i), t\left(\varphi_{\alpha, i}\right)=$ $(s(\alpha), i)$ and with zero differential $\partial$.

Given a box $\mathfrak{A}$, we denote by $\operatorname{rep}(\mathfrak{A})$ the category of finite dimensional representations of $\mathfrak{A}$.

Let us consider the following functor $\mathbf{F}: \operatorname{rep}(\mathfrak{A}(\mathcal{A})) \rightarrow \mathcal{P}_{\text {min }}^{b}(\mathcal{A})$.

A representation $M \in \operatorname{rep}(\mathfrak{A})$ is given by vector spaces $M(x, n)$ and linear mappings $M(\alpha, n): M(y, n) \rightarrow M(x, n-1)$, where $\alpha \in \mathcal{Q}_{1}[x, y]$ and $x, y \in$ $\mathcal{Q}_{0}, n \in \mathbb{Z}$. For such a representation, set $P_{n}=\bigoplus_{x \in \mathcal{Q}_{0}} \mathcal{A}^{x} \otimes M(x, n)$ and $d_{n}=$ $\bigoplus_{x, y \in \mathcal{Q}_{0}} \sum_{\alpha \in \mathcal{Q}[x, y]} \mathcal{A}^{\alpha} \otimes M(\alpha, n)$. A morphism $\Psi: M \rightarrow M^{\prime}$ is given by linear mappings $\Psi(z, n): M(z, n) \rightarrow M^{\prime}(z, n)$ and $\Psi\left(\varphi_{\alpha, m}\right): M(y, n) \rightarrow M^{\prime}(x, n)$, where $x, y, z \in \mathcal{Q}_{0}$ and $\alpha \in \mathcal{Q}_{1}[x, y]$. We define a homomorphism $F(\Psi): F(M) \rightarrow F\left(M^{\prime}\right)$ by the following rule. Given $x, y \in \mathcal{Q}_{0}$ we set

$$
\widetilde{\mathcal{Q}}_{1}[x, y]= \begin{cases}\mathcal{Q}_{1}[x, y] & , \text { if } x \neq y \\ \mathcal{Q}_{1}[x, y] \cup\left\{1_{x}\right\} & , \text { otherwise. }\end{cases}
$$

For given $\alpha \in \widetilde{\mathcal{Q}}_{1}[x, y]$ we set

$$
\Psi_{\alpha, n}= \begin{cases}\Psi\left(\varphi_{\alpha, n}\right) & , \text { if } \alpha \in \mathcal{Q}_{1} \\ \Psi(x, n) & , \text { otherwise. }\end{cases}
$$

Then $F(\Psi)$ is defined by $F(\Psi)_{n}=\bigoplus_{x, y \in \mathcal{Q}_{0}} \sum_{\alpha \in \widetilde{\mathcal{Q}}[x, y]} \mathcal{A}^{\alpha} \otimes \Psi_{\alpha, n}$.

The following Theorem follows from the Theorem 2.2 in BD.

Theorem 2.1. $\mathbf{F}$ is an equivalence of categories.

We define a shift functor in $\operatorname{rep}(\mathfrak{A}(\mathcal{A}))$ by the following rule. Given $M \in$ $\operatorname{rep}(\mathfrak{A}(\mathcal{A}))$ and $j \in \mathbb{Z}$ we define $M[j] \in \operatorname{rep}(\mathfrak{A}(\mathcal{A}))$ by $M[j](a, i)=M(a, i-j)$ and $M[j](\alpha, i)=M(\alpha, i-j)$. In the same way we can define $[j]$ for morphisms in $\operatorname{rep}(\mathfrak{A}(\mathcal{A}))$.

Lemma 2.2. $\mathbf{F}(M[i])=\mathbf{F}(M)[i]$ and $\mathbf{F}(\varphi[i])=\mathbf{F}(\varphi)[i]$ for any object $M$ and any morphism $\varphi$ in $\operatorname{rep}(\mathfrak{A}(\mathcal{A}))$.

Proof. Straightforward.

Given a quiver $\mathcal{Q}$ we fix some vertex $a \in \mathcal{Q}_{0}$ and denote by $\mathcal{Q}[i]$ the connected component of $\mathcal{Q}^{\square}$ which contain the vertex $(a, i)$. Given a walk $w=w_{1} \cdots w_{n}$ in $\mathcal{Q}$ we denote by $\varepsilon^{+}(w)$ (resp., $\varepsilon^{-}(w)$ ) the number of $w_{i}$ of the form $p$ (resp., $\left.p^{-1}\right), p$ being an arrow. We set $\varepsilon(w)=\left|\varepsilon^{+}(w)-\varepsilon^{-}(w)\right|$. We denote by $\mathcal{Q}^{c}$ the set of all closed walks in $\mathcal{Q}$ and set $\varepsilon(\mathcal{Q})=\min _{w \in \mathcal{Q}^{c}} \varepsilon(w)$ in case of $\mathcal{Q}^{c} \neq \emptyset$ and $\varepsilon(\mathcal{Q})=0$ otherwise. We say that a quiver $\mathcal{Q}$ satisfies the walk condition provided $\varepsilon(\mathcal{Q})=0$ (= the number of clockwise oriented arrows is the same as the number of counterclockwise oriented arrows for any closed walk $w$ of $\mathcal{Q}$ ).

Lemma 2.3. Let $b \in \mathcal{Q}_{0}, i, j \in \mathbb{Z}$ and $a$ as above. Then $(b, j) \in \mathcal{Q}[i]$ if and only if there exists a walk $w$ from a to $b$ in $\mathcal{Q}$ such that $j=i+\varepsilon^{+}(w)-\varepsilon^{-}(w)$. 
Proof. Straightforward.

Corollary 2.4. Let $\mathcal{Q}$ be a connected quiver. Then $\mathcal{Q}[i]=\mathcal{Q}[j]$ if and only if there exists a closed walk $w$ in $\mathcal{Q}$ such that $i \equiv j(\bmod \varepsilon(w))$.

Proof. Let $a \in \mathcal{Q}$ be as above.

$" \Longrightarrow$." Suppose that $\mathcal{Q}[i]=\mathcal{Q}[j]$ for some $i \neq j \in \mathbb{Z}$. Then $(a, j) \in \mathcal{Q}[i]$ and by Lemma 2.3 there exists a walk $w$ from $a$ to $a$ in $\mathcal{Q}$ such that $j=i+\varepsilon^{+}(w)-\varepsilon^{-}(w)$, hence $i \equiv j(\bmod \varepsilon(w))$.

$" \Longleftarrow . "$ Let $w$ be a closed walk in $Q$ such that $i \equiv j(\bmod \varepsilon(w))$. Then $j=i+m \varepsilon(w)$ for some $m \in \mathbb{Z}$. Since $\mathcal{Q}$ is connected, there exists a walk $u$ from $a$ to $s(w)$. Then for the closed walk $v=u^{-1} w^{m} u$ in $\mathcal{Q}$ we have $j=i+\varepsilon^{+}(v)-\varepsilon^{-}(v)$. Therefore $(a, j) \in \mathcal{Q}[i]$ by Lemma 2.3 and hence $\mathcal{Q}[j]=\mathcal{Q}[i]$.

Corollary 2.5. Let $\mathcal{Q}$ be a connected quiver. Then $\mathcal{Q}[i]=\mathcal{Q}[j]$ if and only if $i \equiv j(\bmod \varepsilon(\mathcal{Q}))$.

Proof. It is easy to see that if $\mathcal{Q}$ is connected and $\mathcal{Q}^{c} \neq \emptyset$, then for any closed walk $w$ we have $\varepsilon(w)=m \varepsilon(\mathcal{Q})$ for some $m \in \mathbb{N}$. Hence the statement follows from Corollary 2.4.

Lemma 2.6. $\quad 1$. Let $\mathcal{Q}$ be a connected quiver which satisfies the walk condition. Then $\mathcal{Q}^{\square}$ is a disjoint union $\bigsqcup_{i \in \mathbb{Z}} \mathcal{Q}[i]$, where $\mathcal{Q}[i] \simeq \mathcal{Q}^{\text {op }}$ for all $i$.

2. Let $\mathcal{Q}$ be a quiver which not satisfy the walk condition. Then $\mathcal{Q}^{\square}$ is the disjoint union $\bigsqcup_{0 \leq i<\varepsilon(\mathcal{Q})} \mathcal{Q}[i]$, where $\mathcal{Q}[i] \simeq \mathcal{Q}^{\diamond}$ for all $i$ for some quiver $\mathcal{Q}^{\diamond}$.

Proof. $\quad$ 1. It follows from Corollary 2.4 that if $i \neq j$ we have $\mathcal{Q}[i] \neq \mathcal{Q}[j]$. It is easy to see that in this case $\mathcal{Q}[i] \simeq \mathcal{Q}^{\text {op }}$.

2. It is easy to see that $\mathcal{Q}[i] \simeq \mathcal{Q}[j]$ for all $i, j \in \mathbb{Z}$. Therefore the statement follows from Corollary 2.4 .

\section{Derived representation type}

Theorem 3.1. Let $\mathcal{A}$ be a lofd connected category with radical square zero.

1. $\mathcal{A}$ is derived tame if and only if $\mathcal{Q}_{\mathcal{A}}$ is a Dynkin quiver (of types $\mathbb{A}_{n}(n \geq 1)$, $\mathbb{D}_{n}(n \geq 4), \mathbb{E}_{n}(8 \geq n \geq 6)$ ) or an Euclidian quiver (of types $\tilde{\mathbb{A}}_{n}(n \geq 1)$, $\tilde{\mathbb{D}}_{n}(n \geq 4), \tilde{\mathbb{E}}_{n}(8 \geq n \geq 6)$ ) or a quiver of types $\mathbb{A}_{\infty}, \mathbb{A}_{\infty}^{\infty}$ or $\mathbb{D}_{\infty}$.

2. $\mathcal{A}$ is derived discrete if and only if $\mathcal{Q}_{\mathcal{A}}$ is a Dynkin quiver or an Euclidian quiver $\widetilde{\mathbb{A}}_{n}(n \geq 1)$ which does not satisfy the walk condition or a quiver of types $\mathbb{A}_{\infty}, \mathbb{A}_{\infty}^{\infty}$ or $\mathbb{D}_{\infty}$.

3. $\mathcal{A}$ is derived finite if and only if $\mathcal{Q}_{\mathcal{A}}$ is a Dynkin quiver.

Proof. Let $\mathcal{Q}=\mathcal{Q}_{\mathcal{A}}$. We distinguish three cases.

(a) $\mathcal{Q}$ has no cycles.

Then by Lemma 2.6 we have in this case $\mathcal{Q}^{\square}=\bigsqcup_{i \in \mathbb{Z}} \mathcal{Q}[i]$, where $\mathcal{Q}[i]=\mathcal{Q}^{\text {op }}$. Hence the statements of the Theorem in this case follow from $[\mathbf{G}, \mathbf{N}]$ and Proposition 1.1

(b) $\mathcal{Q}$ is an Euclidian quiver $\tilde{\mathbb{A}}_{n}$. 
It follows from Lemma 2.6 that if $\mathcal{Q}$ satisfies the walk condition, then $\mathcal{Q}^{\square}=$ $\bigsqcup_{i \in \mathbb{Z}} \mathcal{Q}[i]$, where $\mathcal{Q}[i]=\mathcal{Q}^{\text {op }}$, hence $\mathcal{A}$ is derived tame, but is not derived discrete by $[\mathbf{N}]$ and Proposition [1.1, and if $\mathcal{Q}$ does not satisfy the walk condition, then by Lemma 2.6 we have $\mathcal{Q}^{\square}=\bigsqcup_{0 \leq i<\varepsilon(\mathcal{Q})} \mathcal{Q}[i]$, where $\mathcal{Q}[i] \simeq \mathbb{A}_{\infty}^{\infty}$ for all $i$, hence $\mathcal{A}$ is derived discrete by $\mathbf{G}$ and Proposition 1.1 .

(c) $\mathcal{Q}$ has an Euclidian sub-quiver $\mathcal{Q}^{\prime} \neq \mathcal{Q}$ of type $\tilde{\mathbb{A}}_{n}$.

It follows from (b) that $\mathcal{Q}^{\prime \square}$ has connected sub-quiver $X$ of type $\tilde{\mathbb{A}}_{n}$ or $\mathbb{A}_{\infty}^{\infty}$. Let $X^{\prime}$ be the connected sub-quiver of $\mathcal{Q}^{\square}$ which contains $X$. Since $\mathcal{Q}^{\prime} \neq \mathcal{Q}$, we have $X^{\prime} \neq X$. Therefore $\mathbb{k} \mathcal{Q}^{\square}$ is wild by $[\mathbf{N}]$ and hence $\mathcal{A}$ is derived wild.

\section{Indecomposable objects}

Let $\mathbf{F}$ be as in Section 2 and $\mathcal{X}$ as in Section 1

Theorem 4.1. Let $\mathcal{A}$ be a lofd category with radical square zero. Then the complexes $\mathbf{F}(M)$ and $\beta(N)$, where $M \in \operatorname{ind} \operatorname{rep}\left(\mathbb{k} \mathcal{Q}^{\square}\right)$ and $N \in \mathcal{X}\left(\mathbb{k} \mathcal{Q}^{\square}\right)$, constitute an exhaustive list of pairwise non-isomorphic indecomposable objects of $\mathcal{D}^{b}(\mathcal{A})$.

Proof. Since $\partial=0$ for the box $\mathfrak{A}=\mathfrak{A}(\mathcal{A})$, we have that ind $\operatorname{rep}(\mathfrak{A})=$ ind $\operatorname{rep}\left(\mathbb{k}^{\square}\right)$. Hence the statement follows from Theorem 2.1 and Proposition 1.1

For a quiver $\mathcal{Q}$ which satisfies the walk condition, we denote by $\imath: \operatorname{rep}\left(\mathcal{Q}^{\text {op }}\right) \rightarrow$ $\operatorname{rep}\left(\mathcal{Q}^{\square}\right)$ the inclusion functor which sent $\mathcal{Q}^{\text {op }}$ to $\mathcal{Q}[0]$. It follows from Lemma 2.6 that in this case gl.dim $\mathcal{A}<\infty$ ( because the quiver $\mathcal{Q}$ has no oriented cycle) and it is the disjoint union $\bigsqcup_{i \in \mathbb{Z}} \mathcal{Q}[i]$, where $\mathcal{Q}[i]=\mathcal{Q}^{\text {op }}$ for all $i$. Hence we obtain the following Corollary.

Corollary 4.2. Let $\mathcal{A}$ be a lofd category with radical square zero whose quiver $\mathcal{Q}=\mathcal{Q}_{\mathcal{A}}$ satisfies the walk condition. Then the complexes $\mathbf{F}(\imath(M))[i]$, where $M \in$ ind $\operatorname{rep}\left(\mathbb{k} \mathcal{Q}^{\text {op }}\right)$ and $i \in \mathbb{Z}$, constitute an exhaustive list of pairwise non-isomorphic indecomposable objects of $\mathcal{D}^{b}(\mathcal{A})$.

\section{References}

[BD] V. Bekkert and Yu. Drozd, Tame-wild dichotomy for derived categories, arXiv:math. $\mathrm{RT} / 0310352$

[BL] R. Bautista and S. Liu, The bounded derived category of an algebra with radical squared zero, Preprint.

[BM] V. Bekkert and H. Merklen, Indecomposables in derived categories of gentle algebras, Algebras and Representation Theory 6 (2003), 285-302.

[D1] Yu. A. Drozd, Tame and wild matrix problems, Representations and quadratic form, Institute of Mathematics, Kiev, 1979, pp. 39-74; English translation: Amer. Math. Soc. Translations 128 (1986), 31-55.

[D2] Yu. A. Drozd, Reduction algorithm and representations of boxes and algebras, Comptes Rendues Math. Acad. Sci. Canada 23 (2001), 97-125.

[D3] Yu. A. Drozd, Derived tame and derived wild algebras, Algebra and Discrete Math. 3 (2004), $57-74$.

[D4] Yu. A. Drozd, Semi-continuity for derived categories, Algebras and Representation Theory 8 (2005), 239-248.

[G] P. Gabriel, Unzerlegbare Darstellungen I, Manuscr. Math. 6 (1972), 71-103.

[GM] S. I. Gelfand and Yu. I. Manin, Methods of Homological Algebra, Springer-Verlag, 1996.

$[\mathrm{H}]$ D. Happel, Triangulated Categories in the Representation Theory of Finite Dimensional Algebras, London Mathematical Society Lecture Notes Series 119, Cambridge University Press, Cambridge, 1988. 
[HR] D. Happel and C. M. Ringel, The derived category of a tubular algebra, Springer LNM 1177 (1984), 156-180.

[N] L. A. Nazarova, Representations of quivers of infinite type, Izv. Akad. Nauk SSSR, Ser. Mat. 37 (1973), 752-791; English transl.: Math. USSR. Izv. 7 (1973), 749-792.

[V] D. Vossieck, The algebras with discrete derived category, J. Algebra 243 (2001), 168-176.

Departamento de Matemática, iCEx, Universidade federal de Minas Gerais, Av. Antônio Carlos, 6627, CP 702, CEP 30123-970, Belo Horizonte-MG, Brasil

E-mail address: bekkert@mat.ufmg.br

Institute of Mathematics, National Academy of Sciences of Ukraine, TereschenKIVSKA 3, 01601 KIEV, UKRAINE

E-mail address: drozd@imath.kiev.ua 